A MORTALITY REVIEW OF CARE HOME RESIDENTS ADMITTED TO ACUTE HOSPITALS

Tomoko Lewis, Maria Debattista, Katherine E Stewart. Lancashire Teaching Hospitals NHS Foundation Trust

10.1136/spcare-2020-PCC.92

Background We are aware that the number of care home residents dying in hospital in our local area is higher than average. We conducted a mortality review focusing on this population to identify areas for improvement. Particular attention was paid to understanding the number of hospital admissions in last 90 days of life, impact of advance care planning (ACP) and barriers to achieving preferred place of death (PPD).

Methods A retrospective review of care home residents who died in hospital over a 3 month period starting on 13.09.18 was undertaken. These patients were known to the hospital palliative care team. Data was collected from the palliative care referral list, electronic patient records and further discussion in MDT.

Results 34 care home residents were admitted and died in hospital. A larger proportion of patients (56\%) were admitted from residential homes compared to nursing homes (38\%). The vast majority of these patients had a non-cancer diagnosis (91\%). 59\% patients had previous emergency admissions in the last 90 days of life and were discharged. 34\% of these were discharged from $A \& E$.

Despite $41 \%$ of patients having a DNACPR in place prior to admission, only $29 \%$ had EPaCCs record created, and only $15 \%$ of patients had a clear PPD documented. Multiple barriers to achieving PPD were highlighted. Results suggested that the most significant were that care homes were unable to meet patients' needs (36\%), rapid deterioration during admission (35\%) and missed opportunities for ACP (21\%). 32\% of these patients changed PPD to hospital, as they were happy with care received.

Conclusions Lack of ACP and communication has been improved with a dedicated palliative care section on hospital discharge letters. Education tailored to acute areas of the trust (A\&E and MAU) is being provided, and increased integrated working in these areas is being explored.

\section{CONTINUOUS SUBCUTANEOUS INFUSION PRESCRIBING PRACTICES IN END OF LIFE CARE: A MULTICENTRED RETROSPECTIVE REVIEW}

Philippa McFarlane, Samuel Raveney, Aruni Wijeratne. Epsom and St Helier Hospital, Princess Alice Hospice

\subsection{6/spcare-2020-PCC.93}

Background Continuous Subcutaneous Infusion (CSCI) prescription practice is an area of high clinical importance across all palliative care settings. Frequent evaluation of CSCI prescribing is essential to ensure appropriate and justified prescriptions are made.

Methods Across three palliative care settings, a hospice and two hospitals (A and B) within a trust, prescriptions of anticipatory (PRN) medications, opioids and continuous subcutaneous infusions (CSCI) of 60 patients who died in April 2019 were reviewed retrospectively.

Results In the hospice anticipatory CSCIs were prescribed for all patients expected to deteriorate with a range given for each medication. This practice was not observed in the hospital setting where it is not recommended to prescribe anticipatory CSCIs. $70 \%$ of the hospice patients had a CSCI in use at the end of life compared to $25 \%$ of the hospital patients. All hospital CSCIs contained midazolam (vs $71 \%$ in the hospice), however the total number of patients receiving midazolam CSCI was higher in the hospice. The numbers of patients dying with a CSCI containing an anti-secretory agent was low in all settings (20\% at hospice, $5 \% \mathrm{~A}, 10 \% \mathrm{~B}$ ). No hospital patients were receiving anti-emetic medication CSCI compared to $57 \%$ of hospice patients. In the hospice, patients died with between $0-5$ medications administered CSCI, compared to $0-3$ in the hospital setting.

Conclusion There were variations in the prescription of CSCIs for patients dying in an acute hospital compared with a hospice. Not only will this likely reflect the varying complexity of patients within the different settings but also staff confidence and competence in recognising and managing symptoms of patients who are at the end of their lives. Patient comfort at the end of life was not evaluated in this audit, review of clinical notes would guide further work.

\section{ADVANCE CARE PLANNING IN HOSPITAL FAST TRACK DISCHARGE PATIENTS: A QUALITY IMPROVEMENT PROJECT}

Laura M Nightingale, Sam Finn. Kingston Hospital NHS Foundation Trust

\subsection{6/spcare-2020-PCC.94}

Introduction There is national focus on earlier identification of patients in their last year of life and allowing them to express their preferences through the process of Advance Care Planning (ACP). Despite training on prognostic models, many clinicians find it difficult to identify which patients are suitable for ACP discussions. Expedited hospital discharges utilising NHS Continuing Care Fast Tracking potentially provide an opportunity to undertake and record ACP, as these patients are anticipated to be approaching the end of life. The aim of this project was to increase the number of ACP discussions in Fast Track discharge patients.

Method This project took place using a Plan, Do, Study, Act approach in three-month iterative cycles.

Fast track discharge patients were identified from databases held by the palliative care and discharge teams. The electronic hospital records of patients identified from both databases were reviewed retrospectively to determine if there was any evidence of ACP. The Co-ordinate My Care (CMC) database was accessed to see if the patient had a CMC record.

Results Overall, the results showed an overall increase in ACP/ CMC from $40 \%$ at baseline to $55 \%$ at 3 months, $98 \%$ at six months, $98 \%$ at nine months and $100 \%$ at 12 months.

This improvement was due to:

- Education and training to junior doctors on ACP/CMC

- Revision of the hospital's ACP proforma to improve quality of information shared

- Simplification of the ACP/CMC process within the hospital resulting in improved communication with external organisations

- Addition of a prompt regarding completion of $\mathrm{ACP} / \mathrm{CMC}$ onto NHS Continuing Care Fast Track tool paperwork 